\title{
DELAY DIFFERENTIAL INCLUSIONS WITH CONSTRAINTS
}

\author{
SHOUCHUAN HU AND NIKOLAOS S. PAPAGEORGIOU
}

(Communicated by Hal L. Smith)

\begin{abstract}
In this paper we examine functional differential inclusions with memory and state constraints. For the case of time-independent state constraints, we show that the solution set is $R_{\delta}$ under Carathéodory conditions on the orientor field. For the case of time-dependent state constraints we prove two existence theorems. For this second case, the question of whether the solution set is $R_{\delta}$ remains open.
\end{abstract}

\section{INTRODUCTION}

Since the pioneering work of Aronszajn [1], several researchers have studied the regularity properties of the solution set of various differential equations and differential inclusions. Recall that a subset of a metric space is called an $R_{\delta}$-set if it is the intersection of a decreasing sequence of nonempty, compact absolute retracts. In [1] it was proved that the solution set of the Cauchy problem $\dot{x}(t)=$ $f(t, x(t)), x(0)=x_{0}$, with $t \in T=[0, r], f(\cdot, \cdot)$ a bounded continuous vector field on $T \times \mathbb{R}^{n}$, is an $R_{\delta}$-set, in particular then is acyclic. Due to the fixed point theorem of Eilenberg-Montgomery [4] for pseudo-acyclic operators, acyclicity is an important property since it can be used to establish the existence of periodic solutions for $\dot{x}(t)=f(t, x(t))$, provided that $f(t, x)$ is periodic in $t$. Aronszajn's results were extended to differential inclusions in $\mathbb{R}^{n}$ by Himmelberg-Van Vleck [9, 10] (autonomous systems) and DeBlasi-Myjak [3] (nonautonomous systems). In a recent paper [11], the authors proved the same regularity result for the solution set of differential inclusions on some $K \subseteq \mathbb{R}^{n}$ (here $K$ is the set of state constraints).

The purpose of the present paper is to study delay differential inclusions with constraints. In case the constraint set $K$ is time independent, we prove that the solution set is an $R_{\delta}$-set and a periodic solution exists if the system is periodic. This way we generalize an earlier result of Haddad-Lasry [7]. When $K$ is timedependent, since the graph of $K$ is not convex in general, we can only establish some existence results and pose as an open problem the question of whether the solution set is an $R_{\delta}$-set.

Received by the editors October 29, 1993.

1991 Mathematics Subject Classification. Primary 34A60, 34K40.

Key words and phrases. Upper semicontinuous multifunction, $R_{\delta}$-set, tangent cone, periodic solution, Hausdorff metric, time-varying constraint. 


\section{Preliminaries}

Let $(\Omega, \Sigma)$ be a measurable space and $X$ a separable Banach space. We define $P_{f(c)}(X)=\{A \subseteq X$ : nonempty, bounded, closed (and convex) $\}$. A multifunction $F: \Omega \rightarrow P_{f}(X)$ is said to be measurable if for all $x \in X$, the $\mathbb{R}_{+}$-valued function $\omega \rightarrow d(x, F(\omega))=\inf \{\|x-y\|: y \in F(\omega)\}$ is measurable.

Let $Y, Z$ be Hausdorff topological spaces and $G: Y \rightarrow 2^{Z} \backslash\{\varnothing\}$. We say that $G(\cdot)$ is upper semicontinuous (u.s.c.) (resp. lower semicontinuous (l.s.c)), if for all $U \subseteq Z$ open, the set $G^{+}(U)=\{y \in Y: G(y) \subseteq U\}$ (resp. $G^{-}(U)=$ $\{y \in Y: G(y) \cap U \neq \varnothing\})$ is open in $Y$.

On $P_{f}(X)$ we can define the Hausdorff metric by

$$
h(A, B)=\max \left[\sup _{a \in A} d(a, B), \sup _{b \in B} d(b, A)\right] .
$$

The metric space $\left(P_{f}(X), h\right)$ is complete and a multifunction $G: X \rightarrow$ $P_{f}(Y)$ is said to be Hausdorff continuous ( $h$-continuous), if it is continuous from $X$ into $\left(P_{f}(Y), h\right)$.

If $K \in P_{f}(X)$ and $x \in K$, then the tangent cone to $K$ at $x$ is defined by

$$
T_{K}(x)=\left\{v \in X: \frac{\lim }{\lambda \downarrow 0} \frac{d(x+\lambda v, K)}{\lambda}=0\right\} .
$$

This is a closed cone and is convex, if $K$ is convex. If $K$ is convex and int $K \neq \varnothing$, then int $T_{K}(x) \neq \varnothing$ also. The normal cone to $K$ at $x$ is defined by

$$
N_{K}(x)=\left\{x^{*} \in X^{*}:\left(x^{*}, x\right)=\sigma\left(x^{*}, K\right)=\sup \left[\left(x^{*}, y\right): y \in K\right]\right\} .
$$

It is well known (see, for example, Aubin-Cellina [2]) that the normal cone is the negative polar cone of $T_{K}(x)$; i.e., $N_{K}(x)=T_{K}(x)^{-}=\left\{x^{*} \in X^{*}:\left(x^{*}, v\right) \leq\right.$ 0 for all $\left.v \in T_{K}(x)\right\}$.

Recall that a set $A \subseteq X$ is contractible, if there exist a continuous $h$ : $[0,1] \times A \rightarrow A$ and $x_{0} \in A$ such that $h(0, x)=x$ and $h(1, x)=x_{0}$. A set $C \subseteq X$ is said to be an absolute retract, if it can replace $\mathbb{R}$ in Tietze's theorem; i.e., for every metric space $Y$ and closed $A \subseteq Y$, each continuous $f: A \rightarrow C$ has an extension $\hat{f}: Y \rightarrow C$. Evidently an absolute retract is contractible. To see this, let $Y=[0,1] \times C$ and $A=\{0,1\} \times C$ as a closed subset of $Y$, and consider on $A$ the map $f(0, x)=x$ and $f(1, x)=x_{0}$ for $x \in C$. Hence an $R_{\delta}$-set is the intersection of compact, contractible sets. In [12] Hyman showed that the converse is also true.

Definition [7]. Let $X, Y$ be two metric spaces. A multifunction $\Gamma: X \rightarrow$ $2^{Y} \backslash\{\varnothing\}$ is said to be $\sigma$-selectionable, if there exists a sequence $\Gamma_{n}: X \rightarrow$ $2^{Y} \backslash\{\varnothing\}$ such that for each $n \geq 1 \Gamma_{n}(\cdot)$ is u.s.c., has a continuous selector and satisfies

(a) $\Gamma_{n+1}(x) \subseteq \Gamma_{n}(x)$ for all $n \geq 1$ and all $x \in X$;

(b) $\Gamma(x)=\bigcap_{n \geq 1} \Gamma_{n}(x)$ for all $x \in X$.

In order to prove the $R_{\delta}$-property of the solution set of differential inclusions in [11], the authors proved the following lemma which is a generalization of Theorem 2.3 of Kisielewicz et al. [13] and will be used in the present paper. 
Let $T=[0, r], X$ and $Y$ be separable Banach spaces, and $K \subseteq X$ be closed. Recall that a function $f: T \times K \rightarrow Y$ is said to be Carathéodory if $t \rightarrow f(t, x)$ is measurable, $x \rightarrow f(t, x)$ is continuous and there exists $\varphi(\cdot) \in L^{1}(T)$ such that $|f(t, x)| \leq \varphi(t)$ a.e.

Lemma 1 [11]. If $f: T \times K \rightarrow Y$ is a Carathéodory function, then for any $\varepsilon>0$ there exists a jointly locally Lipschitz function

$$
f_{\varepsilon}: T \times K \rightarrow Y \text { such that } \int_{0}^{r} \sup _{x \in K}\left\|f(t, x)-f_{\varepsilon}(t, x)\right\| d t \leq \varepsilon .
$$

The following lemma is due to Rybinski [15] and is an extension of Michael's selection theorem.

Lemma 2 [15]. If $G: T \times X \rightarrow P_{f c}(Y)$ is a measurable multifunction and for every $t \in T, G(t, \cdot)$ is l.s.c., then there exists $g: T \times X \rightarrow Y$ such that $t \rightarrow g(t, x)$ is measurable, $x \rightarrow g(t, x)$ is continuous and for all $(t, x) \in T \times X$ we have $g(t, x) \in G(t, x)$.

The following fixed point result is due to Haddad-Lasry [7]:

Lemma 3 [7]. Let $H \subseteq X$ be nonempty, convex, and compact and assume that $\Gamma$ is a $\sigma$-selectionable multifunction from $H$ into $X$ such that $\Gamma(x) \subseteq H$ for all $x \in H$. Then $\Gamma(\cdot)$ has a fixed point in $H$ (i.e., there exists $x \in H$ such that $x \in \Gamma(x))$.

By homology we mean Čech homology with rational coefficients and call a compact metric space $Z$ acyclic if it has the same homology as a one-point space. Let $V, W$ be metric spaces and $T: V \rightarrow 2^{W} \backslash\{\varnothing\}$. We say that $T(\cdot)$ is acyclic if $T(v)$ is compact and acyclic for every $v \in V$. Then $F: V \rightarrow 2^{V} \backslash\{\varnothing\}$ is said to be pseudo-acyclic if $F=\operatorname{roT}$, where $T(\cdot)$ is u.s.c. and acyclic and $r: W \rightarrow V$ is a single-valued, continuous map. The following basic fixed point theorem for pseudo-acyclic maps is due to Eilenberg-Montgomery [4].

Lemma 4 [4]. Let $M$ be an acyclic absolute neighborhood retract (ANR), $N$ a compact metric space, $r: N \rightarrow M$ a continuous single-valued map and $T: M \rightarrow$ $2^{N} \backslash\{\varnothing\}$ an u.s.c. acyclic multifunction. Then $\operatorname{roT}: M \rightarrow 2^{M} \backslash\{\varnothing\}$ has a fixed point (i.e., there exist $x \in M$ such that $x \in(\operatorname{roT})(x))$.

\section{INCLUSIONS WITH CONSTANT CONSTRAINTS}

For a given $\tau>0$ and $\omega>0$, let $J=[-\tau, 0], T=[0, \omega]$, and $C=$ $C\left(J, \mathbb{R}^{n}\right)$. For a nonempty compact and convex $K \subseteq \mathbb{R}^{n}$, define $\mathscr{K}=\{\varphi \in$ $C: \varphi(\xi) \in K$ on $J\}$. Assume that $F: T \times \mathscr{K} \rightarrow 2^{\mathbb{R}^{n}} \backslash\{\varnothing\}$ and $\varphi \in \mathscr{K}$ are given. We consider the following functional differential inclusion with memory:

$$
\left\{\begin{array}{cl}
\dot{x}(t)=F\left(t, x_{t}\right) & \text { a.e. on } T \\
x(\xi)=\varphi(\xi), \quad \xi \in J, & x(t) \in K, \quad t \in T .
\end{array}\right\}
$$

Here $x_{t}(\cdot) \in C$ is defined by $x_{t}(\xi)=x(t+\xi)$. Denote by $S(\varphi)$ the set of all solutions of (1) with initial function $\varphi(\cdot)$ and for $t \in T$ define $S_{t}: \mathscr{K} \rightarrow$ $2^{\mathscr{K}} \backslash\{\varnothing\}$ by $S_{t}(\varphi)=\{x(t+\cdot) \in \mathscr{K}: x \in S(\varphi)\}$. We will show that under some reasonable conditions, $S(\varphi)$ is an $R_{\delta}$-set for every $\varphi \in \mathscr{K}$ and (1) possesses an $\omega$-periodic solution if $F(t, x)$ is $\omega$-periodic in its first variable. 
The basic hypotheses on the data of (1) are the following: $H_{1}: F: T \times \mathscr{K} \rightarrow P_{f c}\left(\mathbb{R}^{n}\right)$ is a multifunction such that

(1) $t \rightarrow F(t, \psi)$ is measurable,

(2) $\psi \rightarrow F(t, \psi)$ is u.s.c.,

(3) $\sup \{\|y\|: y \in F(t, \psi)\} \leq M$ for all $(t, \psi) \in T \times \mathscr{K}$.

$H_{2}: K \subseteq \mathbb{R}^{n}$ is nonempty, compact, and convex.

$H_{3}: F(t, \psi) \cap T_{K}(\psi(0)) \neq \varnothing$ for all $(t, \psi) \in T \times \mathscr{K}$.

Theorem 1. If hypotheses $\mathrm{H}_{1} \rightarrow \mathrm{H}_{3}$ hold, then

(i) for all $\varphi \in \mathscr{K}, S(\varphi)$ is a nonempty $R_{\delta}$-set in $C\left(\widehat{T}, \mathbb{R}^{n}\right)$ with $\widehat{T}=$ $[-\tau, \omega]$,

(ii) $\varphi \rightarrow S(\varphi)$ is $\sigma$-selectionable on $\mathscr{K}$,

(iii) (1) has an $\omega$-periodic solution, if $F(\cdot, x)$ is $\omega$-periodic.

Proof. Without any loss of generality, we may assume that int $K \neq \varnothing$. Indeed, if this is not the case, let $X_{0}=\operatorname{span} K$. This is a subspace of $\mathbb{R}^{n}$ and clearly $K$ has nonempty interior in $X_{0}$. Furthermore, it is easy to see that $T_{K}(x) \subseteq X_{0}$ for all $x \in K$ and the orientor field $F(t, \psi) \cap X_{0}$ satisfies $H_{3}$. Hence we can consider the following problem equivalent to (1):

$$
\left\{\begin{array}{c}
\dot{x}(t) \in F\left(t, x_{t}\right) \cap X_{0} \quad \text { a.e. on } T \\
x(\xi)=\varphi(\xi), \quad \xi \in J, \quad x(t) \in K, \quad t \in T .
\end{array}\right\}
$$

Finally through a translation if necessary, we can always have $0 \in \operatorname{int} K$. Thus there exists $\delta>0$ such that for all $x^{*} \in \mathbb{R}^{n}$

$$
\delta\left\|x^{*}\right\| \leq \sigma\left(x^{*}, K\right)=\sup \left\{\left(x^{*}, x\right): x \in K\right\} .
$$

Invoking Lemma 3 of DeBlasi-Myjak [3], we get multifunctions $F_{0}, G_{n}$ : $T \times \mathscr{K} \rightarrow P_{f c}\left(\mathbb{R}^{n}\right)$ such that: $(1) F_{0}(t, \psi) \subseteq F(t, \psi)$ for all $(t, \psi) \in T \times \mathscr{K}$ and if $\Delta \subseteq T$ is measurable and $\eta: \Delta \rightarrow \mathscr{K}, y: \Delta \rightarrow \mathbb{R}^{n}$ are measurable and $y(t) \in$ $F(t, \eta(t))$ a.e., then $y(t) \in F_{0}(t, \eta(t))$ a.e. on $\Delta ;(2) G_{n}(\cdot, \psi)$ is measurable and $G_{n}(t, \cdot)$ is $h$-continuous; (3) $F_{0}(t, \psi) \subseteq G_{n+1}(t, \psi) \subseteq G_{n}(t, \psi)$ for all $n \geq 1$ and all $(t, \psi) \in T \times \mathscr{K} ;(4) h\left(G_{n}(t, \psi), F_{0}(t, \psi)\right) \rightarrow 0$ as $n \rightarrow \infty$ for all $(t, \psi) \in T \times \mathscr{K} ;(5)\left|G_{n}(t, \psi)\right|=\sup \left\{\|z\|: z \in G_{n}(t, \psi)\right\} \leq M_{1}$ for some $M_{1}>0$ and all $(t, \psi) \in T \times \mathscr{K}$. From property (1) above, it is clear that problem (1) is equivalent to

$$
\left\{\begin{array}{cl}
\dot{x}(t) \in F_{0}\left(t, x_{t}\right) & \text { a.e. on } T \\
x(\xi)=\varphi(\xi), \quad \xi \in J, & x(t) \in K, \quad t \in T .
\end{array}\right\}
$$

In general, we cannot guarantee that the tangential hypothesis $H_{3}$ holds for $F_{0}(t, x)$. Therefore instead of (3), we consider the following approximating problem:

$$
\left\{\begin{array}{cl}
\dot{x}(t) \in G_{n}\left(t, x_{t}\right) & \text { a.e. on } T \\
x(\xi)=\varphi(\xi), \quad \xi \in J, & x(t) \in K, \quad t \in T .
\end{array}\right\}
$$

We claim that

$$
G_{n}(t, \psi) \cap T_{K}(\psi(0)) \neq \varnothing \quad \text { for all }(t, \psi) \in T \times \mathscr{K} .
$$

To this end, given $\varepsilon>0$ by the Scorza-Dragoni theorem, there exist closed $T_{0} \subseteq T$ with $\lambda\left(T \backslash T_{0}\right)<\varepsilon$ (here $\lambda(\cdot)$ is the Lebesgue measure on $T$ ) such that 
$\left.G_{n}\right|_{T_{0} \times \mathscr{K}}$ is $h$-continuous. Since almost all points in $T_{0}$ are points of density, there is a closed $T_{\varepsilon} \subseteq T_{0}$ with $\lambda\left(T_{0} \backslash T_{\varepsilon}\right)<\varepsilon$ such that $T_{\varepsilon}$ contains only points of density of $T_{0}$. Then since $\varepsilon>0$ is arbitrary, it is enough to prove (5) for all $(t, \psi) \in T_{\varepsilon} \times \mathscr{K}$.

Fix $t_{0} \in T_{\varepsilon}$. Note that the multifunction $t \rightarrow F(t, \psi) \cap T_{K}(\psi(0))$ is measurable (cf. Himmelberg [8]). So by the Kuratowski-Ryll Nardzewski selection theorem (see, for example, Himmelberg [8]), we can find $u: T \rightarrow \mathbb{R}^{n}$ measurable such that $u(t) \in F(t, \psi) \cap T_{K}(\psi(0))$ a.e. Thus $u(t) \in F_{0}(t, \psi) \cap T_{K}(\psi(0))$ and so a fortiori $u(t) \in F(t, \psi) \cap T_{K}(\psi(0))$ for all $t \in T \backslash N(\psi)$, for some Lebesguenull set $N(\psi) \subseteq T$. Then there exist $t_{m} \in T_{0} \backslash N(\psi)$ such that $t_{m} \rightarrow t_{0}$ and let $y_{m}=u\left(t_{m}\right) \in G_{n}\left(t_{m}, \psi\right) \cap T_{K}(\psi(0)), m \geq 1$. Upon taking the limit of a convergent subsequence of $\left\{y_{n}\right\}_{m \geq 1}$, since $\left.G_{n}\right|_{T_{0} \times \mathscr{K}}$ is $h$-continuous and $T_{K}(\psi(0))$ is closed, we get for some $y_{0} \in \mathbb{R}^{n}$ that $y_{0} \in G_{n}\left(t_{0}, \psi\right) \cap T_{K}(\psi(0)) \neq \varnothing$. So we have proved (5).

Next for $0<\varepsilon \leq \delta$, define $G_{n}^{\varepsilon}(t, \psi)=G_{n}(t, \psi)+B_{\varepsilon}$, where $B_{\varepsilon}=\left\{x \in \mathbb{R}^{n}\right.$ : $\|x\| \leq \varepsilon\}$. It is obvious that $G_{n}^{\varepsilon}(t, \psi) \cap \operatorname{int} T_{K}(\psi(0)) \neq \varnothing$. Since $G_{n}(t, \psi)$ is measurable in $t$ and $h$-continuous in $\psi$, then $(t, \psi) \rightarrow G_{n}^{\varepsilon}(t, \psi)$ is measurable (cf. Papageorgiou [14]) and so $(t, \psi) \rightarrow G_{n}^{\varepsilon / 3}(t, \psi) \cap T_{K}(\psi(0))$ is measurable. Since $\psi \rightarrow$ int $T_{K}(\psi(0))$ has an open graph (see, for example, Aubin-Cellina [2]), lemma $\beta$ of Flytzanis-Papageorgiou [5] implies that $\psi \rightarrow G_{n}^{e}(t, \psi) \cap$ int $T_{K}(\psi(0))$ is 1.s.c., therefore $\psi \rightarrow \overline{G_{n}^{\varepsilon / 3}(t, \psi) \cap \operatorname{int} T_{K}(\psi(0))}=G_{n}^{\varepsilon / 3}(t, \psi) \cap$ $T_{K}(\psi(0))$ is 1.s.c. Hence according to Lemma 2 , we can find $g_{n}^{1, \varepsilon}: T \times \mathscr{K} \rightarrow \mathbb{R}^{n}$ a function measurable in $t$, continuous in $\psi$ such that

$$
g_{n}^{1, \varepsilon}(t, \psi) \in G_{n}^{\varepsilon / 3}(t, \psi) \cap T_{K}(\psi(0)) \text { for all }(t, \psi) \in T \times \mathscr{K} .
$$

Invoking Lemma 1 , for any $\theta>0$ we can find $g_{n}^{2, \varepsilon}: T \times \mathscr{K} \rightarrow \mathbb{R}^{n}$ which is locally Lipschitz in $(t, x)$ and such that

$$
\int_{0}^{\omega} \sup _{\psi \in \mathscr{K}}\left\|g_{n}^{1, \varepsilon}(t, \psi)-g_{n}^{2, \varepsilon}(t, \psi)\right\| d t<\theta .
$$

It is clear that for each fixed $n \geq 1$, by choosing $\theta>0$ sufficiently small, we can get measurable $A_{n} \subseteq T$ with $\lambda\left(A_{n}\right)<\frac{1}{n^{2}}$ such that

$$
\left\|g_{n}^{1, \varepsilon}(t, \psi)-g_{n}^{2, \varepsilon}(t, \psi)\right\|<\frac{\varepsilon \delta}{3}
$$

for all $(t, \psi) \in\left(T \backslash A_{n}\right) \times \mathscr{K}$. Set $D_{n}=\bigcup_{k=n}^{\infty} A_{k}, \varepsilon=\frac{1}{n}$ and define $G_{n}^{*}(t, \psi)=$ $G_{n}(t, \psi)+B_{1}+\chi_{D_{n}}(t) B_{\beta / \delta}$, where $\beta=\hat{m}+1$ with $\hat{m}=\sup \left\{\left\|g_{n}^{2, \frac{1}{n}}(t, \psi)\right\|\right.$ : $(t, \psi) \in T \times \mathscr{K}\}$.

It is clear that $G_{n}^{*}(t, \psi) \in P_{f c}\left(\mathbb{R}^{n}\right), t \rightarrow G_{n}^{*}(t, \psi)$ is measurable and $\psi \rightarrow$ $G_{n}^{*}(t, \psi)$ is $h$-continuous. We consider

$$
\left\{\begin{array}{cl}
\dot{x}(t) \in G_{n}^{*}\left(t, x_{t}\right) & \text { a.e. on } T \\
x(\xi)=\varphi(\xi), \quad \xi \in J, & x(t) \in K, \quad t \in T .
\end{array}\right\}
$$

Denote the solution set of $(8)_{n}$ by $S_{n}^{*}(\varphi)$. From Haddad [6], we know that $S_{n}^{*} \subseteq C\left(\widehat{T}, \mathbb{R}^{n}\right)$ is nonempty and compact $(\widehat{T}=[-\tau, \omega])$. Also since $\lambda\left(D_{n}\right) \leq$ $\sum_{k=n}^{\infty} \frac{1}{k^{2}} \rightarrow 0$ and for all $(t, \psi) \in T \times \mathscr{K} h\left(G_{n}(t, \psi), F_{0}(t, \psi)\right) \rightarrow 0$ as $n \rightarrow \infty$, we have that $h\left(G_{n}^{*}(t, \psi), F_{0}(t, \psi)\right) \rightarrow 0$ in measure and thus by passing to a 
subsequence if necessary $h\left(G_{n}^{*}(t, \psi), F_{0}(t, \psi)\right) \rightarrow 0$ a.e. Consequently, we have $S_{n+1}^{*}(\varphi) \subseteq S_{n}^{*}(\varphi), n \geq 1$, and

$$
S(\varphi)=\bigcap_{n \geq 1} S_{n}^{*}(\varphi)
$$

Now as in Yorke [16, Theorem 1.3] and in Himmelberg-Van Vleck [10, Proposition 3], we will show that $S_{n}^{*}(\varphi)$ is contractible. Also we will show that $S_{n}^{*}(\cdot)$ admits a continuous selector and so we will have proved the first two conclusions of the theorem.

For each $n \geq 1$, let $g_{n}^{*}(t, \psi)=g_{n}^{2, \frac{1}{n}}(t, \psi)-\frac{1}{3 n} \psi(0)-\frac{\beta}{\delta} \chi_{D_{n}}(t) \psi(0)$, where $g_{n}^{2, \frac{1}{n}}(t, \psi)$ is from (7). Consider the following problem; here $0 \leq s<\omega$ and $\psi \in \mathscr{K}:$

$$
\left\{\begin{array}{c}
\dot{x}(t)=g_{n}^{*}\left(t, x_{t}\right) \\
x(s+\xi)=\varphi(\xi), \quad \xi \in J, \quad x(t) \in K, \quad t \in[s, \omega] . \text { on }[s, \omega]
\end{array}\right\}
$$

We claim that $g_{n}^{*}(t, \psi) \in T_{K}(\psi(0))$ for all $\psi \in \mathscr{K}$. Indeed let $x^{*} \in$ $N_{K}(\psi(0))$ and first assume that $t \notin D_{n}$. Then

$$
\begin{aligned}
\left(x^{*}, g_{n}^{*}(t, \psi)\right)= & \left(x^{*}, g_{n}^{2, \frac{1}{n}}(t, \psi)\right)-\frac{1}{3 n}\left(x^{*}, \psi(0)\right) \\
= & \left(x^{*}, g_{n}^{2, \frac{1}{n}}(t, \psi)-g_{n}^{1, \frac{1}{n}}(t, \psi)\right) \\
& +\left(x^{*}, g^{1, \frac{1}{n}}(t, \psi)\right)-\frac{1}{3 n}\left(x^{*}, \psi(0)\right) \\
\leq & \frac{\delta}{3 n}\left\|x^{*}\right\|-\frac{\delta}{3 n}\left\|x^{*}\right\|=0
\end{aligned}
$$

(recall that $x^{*} \in N_{K}(\psi(0))$ means that $\left(x^{*}, \psi(0)\right)=\sigma\left(x^{*}, K\right)$, hence $\left(x^{*}, \psi(0)\right)$ $\geq \delta\left\|x^{*}\right\|$ from (2)). Thus $g_{n}^{*}(t, \psi) \in T_{K}(\psi(0))$ when $t \notin D_{n}$.

Next let $t \in D_{n}$. Then for $x^{*} \in N_{K}(\psi(0))$ we have

$$
\begin{aligned}
\left(x^{*}, g_{n}^{*}(t, \psi)\right) & =\left(x^{*}, g_{n}^{2, \frac{1}{n}}(t, \psi)\right)-\frac{1}{3 n}\left(x^{*}, \psi(0)\right)-\frac{\beta}{\delta}\left(x^{*}, \psi(0)\right) \\
& \leq\left\|x^{*}\right\|\left(\hat{m}-\frac{\delta}{3 n}-\beta\right) \leq 0 .
\end{aligned}
$$

Therefore, for all $(t, \psi) \in T \times \mathscr{K}$, we have $g_{n}^{*}(t, \psi) \in T_{K}(\psi(0))$. It is clear that $t \rightarrow g_{n}^{*}(t, \psi)$ is measurable, while $\psi \rightarrow g_{n}^{*}(t, \psi)$ is locally Lipschitz. It is then easy to check that $(10)_{n}$ has a unique solution $u_{n}(t ; s, \psi)$ for any $s \in$ $[0, \omega)$ and $\psi \in \mathscr{K}$. Furthermore $(s, \psi) \rightarrow u_{n}(\cdot ; s, \psi)$ is continuous. Also note that $g_{n}^{*}(t, \psi) \in G_{n}^{*}(t, \psi)$ for all $(t, \psi) \in T \times \mathscr{K}$, hence $u_{n}(\cdot ; 0, \psi) \in$ $S_{n}^{*}(\psi)$ and so we have shown that $S_{n}^{*}(\cdot)$ admits a continuous selector. Next we define a homotopy $h:[0,1] \times S_{n}^{*}(\varphi) \rightarrow S_{n}^{*}(\varphi)$ as follows

$$
h(\lambda, x)(t)= \begin{cases}\varphi(t) & \text { if } t \in[-\tau, 0] \\ x(t) & \text { if } t \in[0, \lambda \omega] \\ u\left(t ; \lambda \omega, x_{\lambda \omega}\right) & \text { if } t \in[\lambda \omega, \omega]\end{cases}
$$

This shows that $S_{n}^{*}(\varphi)$ is contractible. 
Finally, we assume that $F(\cdot, \psi)$ is $\omega$-periodic and then prove that (1) has an $\omega$-periodic solution. To this end, let $\widehat{\mathscr{K}}=\left\{\varphi \in A C\left([-\tau, 0], \mathbb{R}^{n}\right):\|\dot{\varphi}(t)\| \leq M\right.$ a.e. $\}$ (here by $A C\left([-\tau, 0], \mathbb{R}^{n}\right)$ we denote the space of absolutely continuous functions on $[-\tau, 0]$ into $\left.\mathbb{R}^{n}\right)$. Let $N=\overline{\bigcup_{\varphi \in \widehat{\mathscr{K}}} S(\varphi)}$. Then $N$ is compact in $C\left(\widehat{T}, \mathbb{R}^{n}\right)$, where $\widehat{T}=[-\tau, \omega]$ (Arzela-Ascoli theorem). Recalling that $S: \widehat{\mathscr{K}} \rightarrow 2^{C\left(\widehat{T}, \mathbb{R}^{n}\right)} \backslash\{\varnothing\}$ is u.s.c. with compact acyclic values (in fact, $R_{\delta}$-values) and noting that $S_{\omega}: \widehat{\mathscr{K}} \rightarrow \widehat{\mathscr{K}}$, we can apply Lemma 4 on the pseudo-acyclic operator $S_{\omega}$ and thus get $\varphi \in S_{\omega}(\varphi)$. Hence there exists $x(\cdot) \in S(\varphi)$ such that $x(\omega+\xi)=x(\xi)$ for every $\xi \in J=[-\tau, 0]$; i.e., this is the periodic trajectory. Q.E.D.

Remarks. (1) Part (iii) of this theorem extends Theorem B.II.1 of Haddad-Lasry [7], who assume that the orientor field $F(t, \psi)$ is jointly u.s.c.

(2) The existence of periodic solutions may also be proved via Lemma 3, but the approach using Lemma 4 that we follow here is much more straightforward.

\section{INCLUSIONS WITH TIME-VARYING CONSTRAINTS}

In this section we assume that $K$ depends on $t$. So let $K: T=[0, \omega] \rightarrow$ $2^{\mathbb{R}^{n}} \backslash\{\varnothing\}, \widehat{K}=\operatorname{gr} K=\left\{(t, x) \in T \times \mathbb{R}^{n}: x \in K(t)\right\}, \mathscr{H}(t)=\{\varphi \in C: \varphi(0) \in$ $K(t)\}$ and $\widehat{\mathscr{H}}=\operatorname{gr} \mathscr{H}=\{(t, \varphi) \in T \times C: \varphi \in \mathscr{H}(t)\}$. Let $F: \widehat{\mathscr{H}} \rightarrow 2^{\mathbb{R}^{n}} \backslash\{\varnothing\}$ and $\left(t_{0}, \varphi\right) \in \widehat{\mathscr{H}}$ with $0 \leq t_{0}<\omega$ be given. We consider the following delay differential inclusion with time varying constraints:

$$
\left\{\begin{array}{c}
\dot{x}(t) \in F\left(t, x_{t}\right) \quad \text { a.e. on }\left[t_{0}, \omega\right] \\
x(\xi)=\varphi\left(\xi-t_{0}\right), \quad \xi \in\left[t_{0}-\tau, t_{0}\right], \quad x(t) \in K(t), \quad t \in\left[t_{0}, \omega\right] .
\end{array}\right\}
$$

In what follows by $D K(t, x)$ we denote the contingent derivative of $K(\cdot)$ at $(t, x) \in \operatorname{gr} K$, i.e., $v \in D K(t, x)(u)$ if and only if $(u, v) \in T_{\widehat{K}}(t, x)$ (cf. Aubin-Cellina [2]).

Theorem 2. Assume that there exist constants $M, M^{\prime}>0$ such that

(1) $K(t) \subseteq B_{M}$ for all $t \in T$ and $t \rightarrow K(t)$ is u.s.c. with closed values,

(2) $F(t, \varphi) \subseteq B_{M}$ on $\widehat{\mathscr{H}}$ and $F(\cdot, \cdot)$ is jointly u.s.c. with closed and convex values.

Then problem (12) above admits a solution for any $\left(t_{0}, \varphi\right) \in \widehat{\mathscr{H}}$ with $t_{0} \in$ $[0, \omega)$ if and only if

$$
F(t, \psi) \cap D K(t, \psi(0))(1) \neq \varnothing
$$

for all $(t, \psi) \in \widehat{\mathscr{H}}$.

Proof. Sufficiency: We follow a well-known procedure; i.e., we transform (12) into a system with constant constraints. To this end, let $\widehat{C}=C\left(J, \mathbb{R}^{1+n}\right)$ and $\mathscr{M}=\left\{\psi^{*} \in \widehat{C}: \psi^{*}(0) \in \widehat{K}\right\}$. Define $G: \mathscr{M} \rightarrow 2^{\mathbb{R}^{1+n}} \backslash\{\varnothing\}$ by

$$
G\left(\psi^{*}\right)=(1, F(t(0), \psi))
$$

for $\psi^{*}=(t(\cdot), \psi(\cdot)) \in \mathscr{M}$, with $t(\cdot) \in C(J, \mathbb{R})$ and $\psi(\cdot) \in C\left(J, \mathbb{R}^{n}\right)$. Note that $G(\cdot)$ is well defined, since $\psi^{*} \in \mathscr{M}$ implies $(t(0), \psi(0)) \in \widehat{K}$, hence $F(t(0), \psi)$ is defined. Let $\varphi^{*}(\xi)=\left(t_{0}+\xi, \varphi(\xi)\right)$ for $\xi \in J$. Then $\varphi^{*} \in \mathscr{M}$. Let $y=(t, x) \in \mathbb{R} \times \mathbb{R}^{n}$. Consider the following autonomous delay 
differential inclusion with constant constraints:

$$
\left\{\begin{array}{cl}
\dot{y}(\sigma) \in G\left(y_{\sigma}\right) \quad \text { on }\left[t_{0}, \omega\right] \\
y(\xi)=\varphi^{*}\left(\xi-t_{0}\right), \quad \xi \in\left[t_{0}-\tau, t_{0}\right], \quad y(\sigma) \in \widehat{K}, \quad \sigma \in\left[t_{0}, \omega\right] .
\end{array}\right\}
$$

It is easy to check that (12) has a solution for $\varphi$ if and only if (15) has a solution for $\varphi^{*}$, where $\varphi^{*}(\xi)=\left(t_{0}+\xi, \varphi(\xi)\right), \xi \in J$. Hence we only need to show that (15) has a solution.

It is clear that $G(\cdot)$ is u.s.c. with compact, convex values. Furthermore, since (13) is equivalent to $(1, F(t, \psi)) \cap T_{\widehat{K}}(t, \psi(0)) \neq \varnothing$, we have for any $\psi^{*} \in(t, \psi) \in \mathscr{M}$,

$$
G\left(\psi^{*}\right) \cap T_{\widehat{K}}\left(\psi^{*}(0)\right) \neq \varnothing .
$$

Consequently, Theorem II-1 of Haddad [6] applies and yields a solution for (15), hence for (12) also.

Necessity: Without any loss of generality, we only prove that (13) is true for $t=t_{0}$. Thus assume that $\left(t_{0}, \varphi\right) \in \widehat{\mathscr{H}}$ is given and the corresponding differential inclusion (12) has a solution. Since $x(\cdot)$ is continuous, $x_{t_{0}}(\cdot)=$ $\varphi(\cdot), F(\cdot, \cdot)$ is u.s.c. at $\left(t_{0}, \varphi\right)$ and $F\left(t_{0}, \varphi\right)$ is compact and convex, for any $\varepsilon>0$ we have that

$$
\frac{x(t)-x\left(t_{0}\right)}{t-t_{0}}=\frac{1}{t-t_{0}} \int_{t_{0}}^{t} \dot{x}(\eta) d \eta \in F\left(t_{0}, \varphi\right)+B_{\varepsilon}
$$

with $t>t_{0}$ sufficiently close to $t_{0}$. Define

$$
V=\left\{y \in \mathbb{R}^{n}: \frac{x\left(t_{n}\right)-x\left(t_{0}\right)}{t_{n}-t_{0}} \rightarrow y \text { for some } t_{n} \downarrow t_{0}\right\} .
$$

It is easy to verify that $(1, V) \subseteq T_{\widehat{K}}\left(t_{0}, \varphi(0)\right)$, hence (17) implies

$$
(1, V) \subseteq\left(1, F\left(t_{0}, \varphi\right)\right) \cap T_{\widehat{K}}\left(t_{0}, \varphi(0)\right) .
$$

Since $x(\cdot)$ is Lipschitzian on $\left[t_{0}, \omega\right]$, we have that $V \neq \varnothing$ and consequently (13) is true with $t=t_{0}$. Q.E.D.

In Theorem 2, we established the existence of a solution for (12) such that $(t, x) \in \widehat{K}$. This suggests that in order to find solutions satisfying a timevarying constraint, maybe $F(\cdot, \cdot)$ need not be defined on all of $\widehat{\mathscr{H}}$, but only on a proper subset of it. In the next theorem we show that indeed this is possible. So to simplify things, assume $t_{0}=0$. Given an initial function $\varphi \in C$, with $\varphi(0) \in K(0)$, we first extend $K$ on $[-\tau, \omega]$ by setting $K(\xi)=$ $\overline{\operatorname{conv}}\{K(0), \varphi(\xi)\}$ for $\xi \in J$. Then it is clear that on $J=[-\tau, 0], K(\cdot)$ is $h$-continuous. For $t \in T$ define $\mathscr{H}_{0}(t)=\{\psi \in C: \psi(\xi) \in K(t+\xi)$ for $\xi \in J\}$ and let $\widehat{\mathscr{H}_{0}}=\operatorname{gr} \mathscr{H}_{0}=\left\{(t, \psi) \in T \times C: \psi \in \mathscr{H}_{0}(t)\right\}$.

Theorem 3. Assume that $F: \widehat{\mathscr{H}_{0}} \rightarrow 2^{\mathbf{R}^{n}} \backslash\{\varnothing\}$ is a jointly u.s.c. multifunction with compact convex values and $F(t, \psi) \subseteq B_{M}$ for all $(t, \psi) \in \widehat{\mathscr{H}}_{0}$. Also assume that $K: T \rightarrow 2^{\mathrm{R}^{n}} \backslash\{\varnothing\}$ is $h$-continuous with compact convex values. Then given $\varphi \in C$ and if for all $(t, \psi) \in \widehat{\mathscr{H}}_{0}, F(t, \psi) \cap D K(t, \psi(0))(1) \neq \varnothing$, problem (12) admits a solution. 
Proof. For $(t, \xi) \in T \times J$, let $P(t, \xi)$ be the metric projection from $\mathbb{R}^{n}$ to $K(t+\xi)$. Since $K(\cdot)$ has compact convex values, this map is single valued and nonexpansive. Also define the operator $\mathscr{P}(t): \mathscr{H}(t) \rightarrow \mathscr{H}_{0}(t)$ by $(\mathscr{P}(t) \psi)(\xi)=$ $P(t, \xi) \psi(\xi)$. We have $\mathscr{P}(t) \psi \in \mathscr{H}_{0}(t)$ (recall that $\left.K\right|_{[-\tau, 0]}$ is $h$-continuous). Define $G: \widehat{H} \rightarrow 2^{\mathbb{R}^{n}} \backslash\{\varnothing\}$ by $G(t, \psi)=F(t, \mathscr{P}(t) \psi)$ (this is well defined since $\mathscr{P}(t) \psi \in \mathscr{K}_{0}(t)$ for every $\left.(t, \psi) \in T \times C\right)$. It is easy to see that $G(\cdot, \cdot)$ is u.s.c. and has compact and convex values. Moreover, for $(t, \psi) \in \widehat{\mathscr{H}}$, since $(\mathscr{P}(t) \psi)(0)=P(t, 0) \psi(0)=\psi(0)$, we get from the tangential hypothesis that

$$
G(t, \psi) \cap D K(t, \psi(0))=F(t, \mathscr{P}(t) \psi) \cap D K(t,(\mathscr{P}(t) \psi)(0)) \neq \varnothing .
$$

Therefore we can apply Theorem 2 to obtain a solution $x(\cdot)$ for

$$
\left\{\begin{array}{c}
\dot{x}(t) \in G\left(t, x_{t}\right) \quad \text { a.e. on } T=[0, \omega] \\
x(\xi)=\varphi(\xi), \quad \xi \in J=[-\tau, 0], \quad x(t) \in K(t), \quad t \in T .
\end{array}\right\}
$$

In particular, $\dot{x}(t) \in F\left(t, \mathscr{P}(t) x_{t}\right)$ a.e., $\mathscr{P}(t) x_{t}=P(t, \xi) x(t+\xi)$ and $x(t+\xi) \in K(t+\xi)$ for all $(t, \xi) \in T \times J$. Hence $\mathscr{P}(t) x_{t}=x_{t}$ for $t \in T$ and consequently, $x(\cdot)$ solves (12). Q.E.D.

Remark. In general, $\widehat{K}$ is not convex, hence the arguments in the proof of Theorem 1 are not applicable when time-dependent constraints are present. So the question of whether the solution set of (12) is an $R_{\delta}$-set remains open.

\section{REFERENCES}

1. N. Aronszajn, Le corespondant topologique de l'unicité dans la theorie des equations differentielles, Ann. of Math. 43 (1942), 730-738.

2. J.-P. Aubin and A. Cellina, Differential inclusions, Springer-Verlag, Berlin, 1984.

3. F. S. DeBlasi and J. Myjak, On the solution set for differential inclusions, Bull. Polish Acad. Sci. 33 (1985), 17-23.

4. S. Eilenberg and D. Montgomery, Fixed point theorems for multivalued transformations, Amer. J. Math. 68 (1946), 214-222.

5. E. Flytzanis and N. S. Papageorgiou, Existence of monotone and slow solutions for differential inclusions, Internat. J. Systems Sci. 20 (1989), 2241-2249.

6. G. Haddad, Monotone trajectories of differential inclusions and functional differential inclusions with memory, Israel J. Math. 39 (1981), 83-100.

7. G. Haddad and J.-M. Lasry, Periodic solutions of functional differential inclusions and fixed points of $\sigma$-selectionable correspondences, J. Math. Anal. Appl. 96 (1983), 295-312.

8. C. J. Himmelberg, Measurable relations, Fund. Math. 87 (1975), 59-71.

9. C. J. Himmelberg and F. S. Van Vleck, On the topological triviality of the solution sets, Rocky Mountain J. Math. 10 (1980), 247-252.

10. (1982), 621-625.

11. S. Hu and N. S. Papageorgiou, On the topological regularity of the solution set of differential inclusions with constraints, J. Differential Equations 107 (1994), 280-289.

12. D. Hyman, On decreasing sequences of compact absolute retracts, Fund. Math. 64 (1969), 91-97.

13. M. Kisielewicz, Z. Nowak, and M. Przybybowska, An approximation theorem for vector valued functions, Funct. Approx. Comment. Math. XII (1982), 55-62.

14. N. S. Papageorgiou, On measurable multifunctions with applications to random multivalued equations, Math. Japon. 32 (1987), 437-464. 
15. L. Rybinski, On Carathéodory type selections, Fund. Math. 125 (1985), 187-193.

16. J. Yorke, Spaces of solutions, Lecture Notes in Oper. Res. and Math., vol. 12, SpringerVerlag, Berlin, 1969, pp. 383-403.

Department of Mathematics, Southwest Missouri State University, SPringfield, MisSOURI 65804

Department of Mathematics, National Technical University, Zografou Campus, ATHENS 15773, GREECE

Current address: Department of Applied Mathematics, Florida Institute of Technology, 150 West University Boulevard, Melbourne, Florida 32901-6988 